\title{
EDITORIAL
}

\section{What do the neurosciences tell us about anxiety disorders? A comment ${ }^{1}$}

Defensive responses range from simple reflex withdrawal and accompanying physiological reactions present from invertebrates to humans, to far more intricate emotional behaviour typical of higher taxa. Is there some order in the numerous circuits and neuroregulators that are implicated in the acquisition and expression of many different forms of defensive behaviour across phyla and in the human clinical syndromes in which they appear? The sheer complexity of the problem has defied attempts to integrate such knowledge with that of the various anxiety syndromes in meaningful detail. The task, however, is no longer altogether hopeless.

The same four basic strategies of defensive behaviour - withdrawal, immobility, aggressive defence, and deflection of attack (Marks, 1986) - are found in both invertebrates and vertebrates, although only withdrawal and immobility seem to be involved in phobic and obsessive-compulsive disorders. Also common across phyla are the basic forms of learning such as habituation, sensitization, and classical and instrumental conditioning.

Some insights have come from animal studies of forms of behaviour like passive and active avoidance, escape, freezing, immobility and startle. Further knowledge has accrued from experiments with forms of defensive learning involving classical conditioning, sensitization and habituation. Information about such behaviour and processes has aided our understanding of the modes of action of behavioural treatment (Marks, 1986) and anxiolytic drugs (Gray, 1982).

It is, of course, a cliché that similar forms of behaviour have repeatedly evolved across taxa independently of one another by different mechanisms (convergence). On the other hand, conservation of evolutionary mechanisms has been found repeatedly. Is there any reason to believe that some of the shared defensive strategies and ways in which they are modified by learning are mediated by common physiological mechanisms? Are there enough points in common between invertebrates and vertebrates to make this likely? Some organizing principles are, in fact, emerging.

First, in vertebrates 50 years of research have produced convincing evidence that several deep structures in the brain are involved in fearful withdrawal and immobility. Modification of withdrawal in both aplysia and mammals is mediated by changes not at the peripheral sensory receptor in skin or muscle, but central to the body of the sensory neuron - among others, at the synapse of the sensory neuron with an interneuron and/or a motor neuron in aplysia, such synapses being in the spinal cord and brain in mammals. The deep mammalian structures related to fear behaviour include the limbic system, hypothalamus, some thalamic nuclei, and some brainstem and cerebellar nuclei. We do not yet understand the role of the neocortex beyond its possible contribution to cognitive aspects of fear that are finally expressed through the deep structures.

Tentative circuits have been sketched that are implicated in some varieties of withdrawal and immobility, some being known in more detail than others (reviewed by Gray, 1982; and Marks, 1986). We shall oversimplify. Escape is partly mediated by circuits through the peri-aqueductal grey matter, some hypothalamic nuclei and amygdala. The substrate for active avoidance is obscure, but may have some relationship to activity in the cingulate cortex. Passive avoidance is controlled by the septo-hippocampal system and afferents to it from the locus coeruleus and rostral raphe nuclei in the brainstem; the amygdala may also be relevant. Tonic immobility is influenced by median raphe nuclei in the brainstem and their efferents. Circuits for freezing and for startle are unclear.

\footnotetext{
1 This paper is adapted from a chapter in Fears, Phobias and Rituals, by I. M. Marks, Oxford University Press: New York (1986). Address for correspondence: Professor 1. M. Marks, Institute of Psychiatry, De Crespigny Park, Denmark Hill, London SE5 8AF.
} 
Secondly, in the last 15 years knowledge about neurotransmitters and neuromodulators has refined our understanding of the relevant circuits. Impressive correspondence has been found between distributions of particular compounds (noradrenalin, serotonin, GABA and neuropeptides) and of pathways and structures mediating forms of defensive behaviour. Study of such compounds in simpler invertebrate nervous systems has produced data relevant to elementary forms of learning that modify normal and abnormal defensive behaviour.

In aplysia gill withdrawal followed by immobility is sensitized by serotonergic stimulation (Kandel, 1983). Related effects of serotonin are seen in vertebrates, including mammals: (i) serotonin increases tonic immobility (Gallup \& Maser, 1977); (ii) it probably facilitates freezing in rats (Driscoll \& Battig, 1982); (iii) it definitely enhances the suppression by punishment of behaviour during approach in conflict situations (Graeff, 1981; Sepinwall, 1983). The same neurotransmitter serotonin - consistently augments immobility and suppression of behaviour across phyla. Such evidence supports Kandel's (1984) suggestion that serotonergic systems sensitize those defensive responses which involve cessation of ongoing activity. However, there are as yet no consistent data to show that any clinical form of anxiety is increased by serotonergic agonists or reduced by serotonergic antagonists.

In mammals noradrenergic systems can play a parallel activating role, but more for alarm with increased motor and autonomic activity rather than for immobility and freezing. There is mammalian evidence of this for the locus coeruleus and fibres ascending from it (Redmond \& Huang, 1979), but data about such systems in invertebrates are lacking. In normals and in anxious patients substances that increase noradrenergic function raise anxiety, and the converse applies for drugs that reduce it (Charney et al. 1984).

An intriguing feature of the two best-known defense-activating systems (serotonergic and noradrenergic) is that their nuclei of origin are in an area as old as the brainstem (locus coeruleus and raphe) with their long projections spreading up and down for a considerable distance. The nuclei are in a region where they could easily be activated by sensory inputs and then, depending on the type of input, activate relevant forebrain structures that regulate defensive outputs. This might be part of the function of the reticular activating system. Recent evidence suggests that there are complex, but still obscure, interactions between the serotonergic and noradrenergic systems.

It is just possible that there are additional defence-activating systems. One could be dopaminergic from the ventral tegmentum to frontal cortex (Gray, 1982; Iversen, 1985). Dopaminergic mechanisms seem to be involved in the action of the anxiolytic buspirone (Taylor et al. 1983); dopaminergic turnover in frontal cortex rises during the immobility of rats that can be conditioned to unavoidable foot-shock and blocked by benzodiazepines (Reinhard et al. 1982). There might perhaps also be endogenous anxiogenic compounds acting on benzodiazepine (BZ) receptors, e.g. some betacarbolines and purines (Sepinwall, 1983; Haefely, 1983).

In contrast to the focal fear-activating systems with their long projections are the diffuse inhibiting short GABA-ergic circuits and associated BZ receptors present throughout the brain and spinal cord. These seem to act by modulating the ascending activating systems (serotonergic, noradrenergic and probably dopaminergic) rather than by directly inhibiting the deep structures that express fear. Certain anxiolytic drugs, like the benzodiazepenes, seem to act in this indirect fashion by potentiating the GABA-ergic and BZ receptor complex.

Another mechanism which attenuates defensive behaviour appears to involve neuropeptides, including endogenous opiates (Post et al. 1984), which are mainly known as inhibitors of pain. Noxious stimuli can decrease pain, apparently through the production of opiates acting on pain pathways. Fear probably does the same, fear taking priority over pain (Bolles \& Fanselow, 1980). Conversely, long-term production of endogenous opiates could inhibit ascending fear pathways, thus reducing fear. Opiates could act like the other (GABA-ergic) inhibiting system through short circuits present diffusely in the nervous system.

Habituation is the attenuation of responses by repeated stimulation. It is found across phyla and has been especially well described with defensive behaviour. The chief treatment to reduce phobias and rituals - exposure - has many characteristics of habituation. Could fear habituation be 
mediated by the two known inhibiting systems - GABA-BZ complexes and opiates? The GABA-BZ complex is not a good candidate, as the reduction of fear in animals and in man by $\mathrm{BZ}$ drugs or alcohol does not last and large doses may actually impair habituation from exposure (Gray, 1982; Marks, 1986). As stress continues, both opiate production and fearful responses diminish - perhaps these reflect the same process?

Another possibility stems from the fact that repeated stress in rodents leads to widespread down-regulation of central beta-adrenergic postsynaptic receptors; this correlates closely with reduction in behavioural responses to repeated stress (Stone, 1983). The same receptors are down-regulated by most known antidepressants (Charney et al. 1981; Snyder \& Peroutka, 1984), which are anxiolytic for some phobics and obsessive-compulsives (Marks, 1983). The possibility thus arises that both a psychological treatment (exposure) and drug treatment may act on the same receptors. It would be interesting to test this notion in simpler organisms like invertebrates in which habituation of withdrawal and of threat to repeated stimulation has been extensively described (reviewed by Kandel, 1984; Marks, 1986).

Pathological anxiety might result from a malfunction of the mechanisms involved in sensitization and/or habituation of fear-anxiety and other defensive responses. Anxiety could rise through a direct or indirect increase in sensitizing mechanisms, and the converse should apply with habituating mechanisms. Sensitizing and habituating mechanisms interact.

Sensitization is relatively non-specific. Perhaps we could equate generalized anxiety with pathological sensitization? Is it merely facile to compare the enduring agitation of sensitized snails (Kandel, 1983) to the motor equivalents seen in an anxiety state (generalized anxiety disorder and panic disorder) and in many agoraphobics?

Unlike sensitization, habituation is specific, and this is mirrored by the specificity of exposure. Response decrement during habituation is found only in those pathways that have been repeatedly stimulated, just as specific reduction in fear of particular phobia- and ritual-evoking situations is seen as exposure is directed at them. Little generalization is seen either with habituation or with exposure. We cannot argue automatically from therapy to aetiology, but it is tempting to suppose that phobias and compulsive rituals were acquired and/or maintained with the same specificity that is needed for their reduction by exposure and, further, that they might be linked to specific failures of habituation. On the other hand, we saw that known fear-attenuating mechanisms (GABA-BZ, opiate and maybe others) are widely distributed in the brain.

If we mapped out the overlapping and distinctive clinical features and treatment requirements of various forms of anxiety disorder we would obtain a series of interlocking Venn diagrams (Marks, 1986). Despite the considerable overlap, there seems to be some specificity of mechanism for each disorder. Some of these specificities may derive from the differing behavioural components (freezing, startle, escape, passive and active avoidance) which appear in various anxiety disorders. Attempts to match such behaviour with clinical features yield some plausible analogies for anxiety-reduction by behavioural treatment, but shed less light on the origins and maintenance of anxiety (Mineka, 1985).

Is the study of brain mechanisms more illuminating? Some forms of anxiety disorder might, in theory, be triggered at specific points of the various circuits mediating different aspects of anxiety. Could obsessive-compulsive disorder originate in a failure of a normal 'checking' function of a comparator somewhere in the septo-hippocampal system (Gray, 1982)? Are the spontaneous panics of agoraphobia and anxiety states massed (?epileptic) discharges of the central noradrenergic neurons in the locus coeruleus and maybe serotonergic neurons nearby, precipitated perhaps by unknown metabolic changes (Haefely, 1983)? The stuttering onset of anxiety and panic is reminiscent of how intermittent stimulation of the amygdala kindles epilepsy (Marks, 1986). Perhaps such malfunctions could produce the types of withdrawal and of immobility seen in anxiety syndromes.

More precise links are required between such potential malfunctions with resultant defensive responses of the kinds found with phobias and rituals, and the ways in which they can be sensitized, conditioned and habituated. The attenuation of fear-anxiety by exposure treatment and by drugs need not necessarily be operating by direct correction of such malfunctions; they could act via more 
indirect processes. If so, better knowledge of those malfunctions might lead to the development of more direct and even more potent treatments. Whatever physiological dysfunctions are eventually found, however, they are not likely to be simple panic buttons. More probably, they are complex disturbances meshing with genetic, developmental and traumatic and vicarious learning effects.

ISAAC MARKS AND ADOLF TOBEÑA

Thanks are due to Iain Campbell, Jeffrey Gray and Susan Iversen for valuable comments on the manuscript.

\section{REFERENCES}

Bolles, R. C. \& Fanselow, M. S. (1980). A perceptual-defensiverecuperative model of fear and pain. Behavioral and Brain Sciences 3, 291-323.

Charney, D., Menkes, D. B. \& Henninger, G. R. (1981). Receptor sensitivity and the mechanisms of action of antidepressant treatment: implications for the etiology and therapy of depression. Archives of General Psychiatry 38, 1160-1180.

Charney, D., Henninger, G. R. \& Breier, A. (1984). Noradrenergc function in panic anxiety: effects of yohimbine in healthy subjects and patients with agoraphobia and panic disorder. Archives of General Psychiatry 41, 751-763.

Driscoll, P. \& Battig, K. (1982). Behavioral, emotional and neurochemical profiles of rats selected for extreme differences in active, two way avoidance, performances. In Genetics of the Brain (ed. I. Lieblich), pp. 95-123. Elsevier: Amsterdam.

Gallup, G. G. \& Maser, J. D. (1977). Tonic immobility. In Psychopathology: Experimental Models (ed. J. Maser and M. Seligman), pp. 334-357. W. H. Freeman: San Francisco.

Graeff, G. G. (1981). Minor tranquilizers and brain defense systems. Brazilian Journal of Medicine and Biological Research 14, 239-265.

Gray, J. A. (1982). The Neuropsychology of Anxiety: an Enqiry into the Functions of the Septohippocampal System. Oxford University Press: Oxford.

Haefely, W. (1983). The biological basis of benzodiazepine action. Journal of Psychoactive Drugs, 1-32.

Iversen, S. D. (1985). Where in the central nervous system do benzodiazepines act? In Psychopharmacology-Progress and Future Prospects (ed. S. D. Iversen), pp. 75-88. Oxford Unıversity Press: Oxford.

Kandel, E. R. (1983). From metapsychology to molecular biology: explorations into the nature of anxiety. American Journal of Psychiatry 140, 1277-1293.

Kandel, E. R. (1984). Steps toward a molecular grammar for learning: explorations into the nature of memory. Harvard/Bicentennial Symposium.

Marks, I. M. (1983). Are there anticompulsive or antiphobic drugs? Review of the evidence. British Journal of Psychiatry 143, 338-347.

Marks, I. M. (1986). Fears, Phobias and Rituals. Oxford University Press: New York (in the press).

Mineka, S. (1985). Animal models of anxiety based disorders. In Anxiety and the Anxiety Disorders (ed. J. Maser and H. Tuma). Lawrence Erlbaum: Hillsdale, NJ.

Post, R. M., Pickar D., Ballenger, J. C. M., Naber, D. \& Rubinow, D. R. (1984). Endogenous opiates in cerebro-spinal fluid: relationship to mood and anxiety. In Neurobiology of Mood Disorders (ed. R. M. Post and J. C. Ballenger), pp. 356-368. Williams \& Wilkins: Baltimore.

Redmond, D. E. \& Huang, Y. H. (1979). New evidence for a locus coeruleus norepinephrine connection with anxiety. Life Sciences 25, 2149-2162.

Reinhardt, J. F., Bannon, M. J. \& Roth, R. H. (1982). Acceleration by stress of dopamine synthesis and metabolism in prefrontal cortex : antagonism by diazepam. Archives of Pharmacology (new series) 318, 374-377.

Sepinwall, J. (1983). Behavioural studies related to the neurochemical mechanisms of action of anxiolytics. In Anxiolytics: Neurochemical, Behavioral and Clinical Perspectives (ed. J. B. Malick, S. J. Enna and H. I. Yamamura). Raven Press: New York.

Snyder, S. H. \& Peroutka, S. J. (1984). Antidepressants and neurotransmitter receptors. In Neurobiology of Mood Disorders (ed. R. M. Post and J. C. Ballenger), pp. 686-697. Williams \& Wilkins: Baltimore.

Stone, E. A. (1983). Problems with current catecholamine hypothesis of antidepressant agents: speculations leading to a new hypothesis. Behavioral and Brain Sciences 6, 535-577.

Taylor, D. P., Riblet, L. A. \& Stanton, H. C. (1983). Dopamine and anxiolytics. In Anxiolytics: Neurochemical, Behavioral and Clinical Perspectives (ed. J. B. Malick, S. J. Enna and H. I. Yamamura). Raven Press: New York. 